\title{
Virtual tours to the KATRIN experiment
}

\section{Manuel Klein, ${ }^{a,}{ }^{*}$ Christian Humm, ${ }^{b}$ Leonard Köllenberger ${ }^{a}$ Philipp Niemann,,${ }^{b, c}$ Yannic Scheuermann, ${ }^{c}$ Philipp Schrögel ${ }^{b}$ and Kathrin Valerius ${ }^{a}$}

${ }^{a}$ Karlsruhe Institute of Technology (KIT), Institute for Astroparticle Physics (IAP), Hermann-von-Helmholtz-Platz 1, 76344 Eggenstein-Leopoldshafen, Germany

${ }^{b}$ Karlsruhe Institute of Technology (KIT), Department for Science Communication, Englerstraße 2, 76131 Karlsruhe, Germany

${ }^{c}$ National Institute for Science Communication (NaWik),

Englerstraße 2, 76131 Karlsruhe, Germany

E-mail: manuel.klein@kit.edu, christian.humm@kit.edu,

leonard.koellenberger@kit.edu, niemann@nawik.de, scheuermann@nawik.de, philipp.schroegel@kit.edu, kathrin.valerius@kit.edu

The KArlsruhe TRItium Neutrino (KATRIN) experiment performs a model-independent measurement of the electron neutrino mass with a design sensitivity of $0.2 \mathrm{eV} / \mathrm{c}^{2}$ (90\% C.L.) after three full years of measurement time. KATRIN measures near the endpoint of the tritium beta spectrum, using the MAC-E filter principle, and operates a $70 \mathrm{~m}$ long beamline. Its technological challenges include the high-luminosity tritium source, the cryogenic pumping section and the $23 \mathrm{~m}$ long ultra-high vacuum vessel of the main spectrometer.

Guided tours to the KATRIN beamline with supporting presentations are frequently offered to make the experiment, astroparticle physics and scientific research in general accessible to the public and to students in particular. However, the on-site access is limited by the operation of high voltage and magnets, safety regulations for the tritium laboratory and the ongoing pandemic. This fuelled the development of three kinds of virtual tours: a 40-minute-long video tour with live commentary via zoom was created using cellphone-made footage of the beamline and archive footage of the transport and commissioning of the key components; a 3D VR panorama of five locations at the beamline for virtual reality headsets or as browser application providing a live-action guide or free exploration was developed with the National Institute for Science Communication (NaWik); and a browser interface for a low-poly model of the full beamline is work in progress. In this contribution, we present all three tours and their making, including comparative NaWik research on the knowledge transfer potential of virtual presentation forms.

Supported by BMBF (Ø05A20VK3), the Helmholtz Association, the Klaus Tschira Foundation, the KIT centre KCETA, and the Excellence Strategy of the German Federal and State Governments.

$37^{\text {th }}$ International Cosmic Ray Conference (ICRC 2021)

July 12 th - 23rd, 2021

Online - Berlin, Germany

\footnotetext{
*Presenter
} 


\section{Contents}

1 The KArlsruhe TRItium Neutrino (KATRIN) experiment 2

2 Motivation for virtual tours 3

3 Three kinds of virtual tours $\quad 4$

3.1 Guided video tours 4

$3.2360^{\circ}$ VR panorama

3.3 Low-poly model of the detector section 6

4 Reception study by the National Institute for Science Communication (NaWik) 6

5 Summary and conclusion $\quad 7$

\section{Introduction}

Virtual presentations offer exceptional potential for scientific outreach beyond conventional slides and posters: complex experimental setups and physical processes can be displayed dynamically and enable an interactive approach to gain an intuitive understanding. In order to harness this potential for the KATRIN experiment, three kinds of virtual tours are being pursued: a guided video tour, a $360^{\circ} \mathrm{VR}$ panorama, and a 3D viewer for a low-poly model.

In this proceeding, the KATRIN experiment is introduced first. Then the motivation for virtual tours is discussed, before all three kinds of tours are presented. As detailed afterwards, one of these tours was co-created by the National Institute for Science Communication (NaWik), which performs comparative research on the reception of various presentation forms. A summary and conclusions mark the end.

\section{The KArlsruhe TRItium Neutrino (KATRIN) experiment}

The KArlsruhe TRItium Neutrino (KATRIN) experiment performs a model-independent measurement of the electron neutrino mass with a design sensitivity of $0.2 \mathrm{eV} / \mathrm{c}^{2}$ (90\% C.L.) after three full years of measurement time [1]. KATRIN measures the tritium beta spectrum near its kinematic endpoint with a MAC-E filter (Magnetic Adiabatic Collimation of the beta electrons and an Electrostatic retarding potential) [2,3], which is realised via the $70 \mathrm{~m}$ long beamline [4]. Its technological challenges include (see figure 1):

A) the high-luminosity tritium source, which provides $10^{11}$ beta decays per second and can be cooled down to $30 \mathrm{~K}$; 


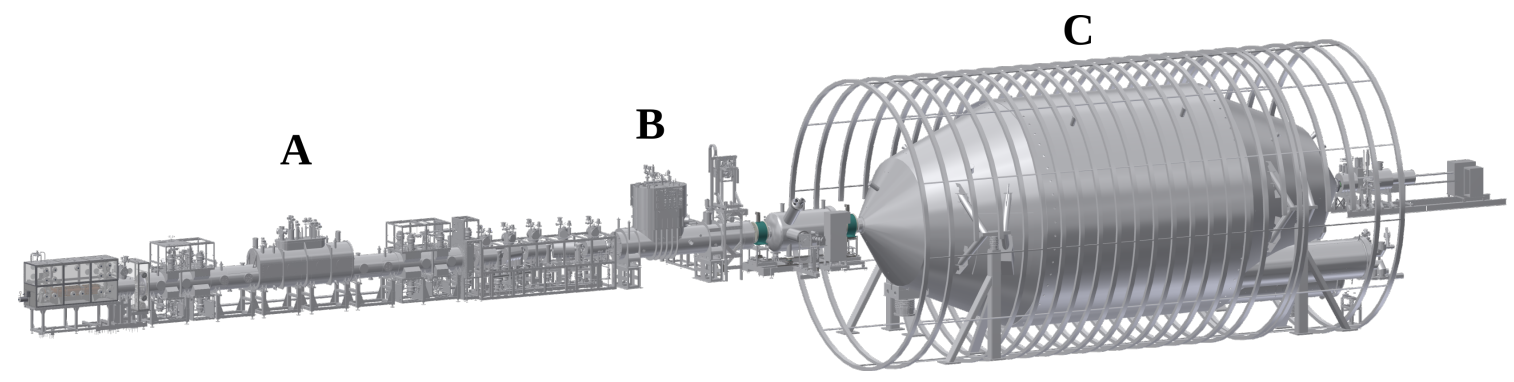

Figure 1: Overview of the $70 \mathrm{~m}$ long KATRIN beamline. Major technological challenges include the high-luminosity tritium source (A), the cryogenic pumping section (B), and the main spectrometer (C).

B) the cryogenic pumping section at $3 \mathrm{~K}$;

C) the main spectrometer with an ultra-high vacuum vessel of $23 \mathrm{~m}$ length and $10 \mathrm{~m}$ diameter, to which a high voltage of typically $-18.6 \mathrm{kV}$ is applied with a precision of $2 \mathrm{ppm}$;

D) superconducting solenoids, which provide a magnetic guiding field between $2.7 \mathrm{G}$ and $6 \mathrm{~T}$ with a typical strength of several Tesla.

Following the Design report in 2004 [1], the beamline was set up step by step for the start of tritium measurements by 2018. Already one year later, in 2019, a new upper limit on the absolute neutrino mass scale was obtained after only four weeks of measurement [5]. In 2021, the first sub-eV result of $m_{v}<0.8 \mathrm{eV} / \mathrm{c}^{2}(90 \%$ C.L.) was released [6].

\section{Motivation for virtual tours}

Guided tours to the KATRIN beamline are offered frequently to make the experiment, astroparticle physics and scientific research in general accessible to the public and to students in particular. The location at KIT Campus North close to the city of Karlsruhe is easy to reach and access to the campus is possible with small organisation effort. On site, the guided tours are supported by posters or - less commonly - slide-based presentations.

Although these lab visits are very well-received and cause little effort in the execution, four major reasons motivated the additional development of virtual tours:

- Travel restrictions. During the COVID-19 pandemic, on-site visits were impeded by official travel restrictions. However, feedback to the virtual tours showed that they also benefit interested laypeople who would not travel to the experiment otherwise.

- Access restrictions at the beamline. During nominal measurement operation, access to most of the beamline is restricted due to the extreme experimental conditions. Due to the radioactivity in the source, safety regulations require an extra amount of preparation to visit the source and transport sections, which make up about half of the beamline. Further, the high voltage operation at the main spectrometer and magnetic fields from superconducting magnets require to keep distance to the beamline. Eventually, the closed vacuum setup restricts the view on the interior features, such as the electrode system inside the spectrometers. 
- Visualisation. The aforementioned restrictions can be overcome with photos even on classical slides and posters. One advantage over on-site visits is that pictures allow to show the whole beamline, which is spread over two separate buildings. But in terms of immersive experience, static images fall short compared to virtual reality. And with videos, it is possible to show past events (like the transport of the main spectrometer vessel) or visualise complex dynamic processes (like the measurement principle of the MAC-E filter).

- Interactivity. Virtual reality allows to interact with the experiment and gain an intuitive understanding. For example, large-scale components of the beamline can be (dis)assembled, the measurement conditions can be changed and the laboratory environment can be explored freely. Neither of that is possible for a visitor on site.

To satisfy this broad range of motivations, three different types of virtual tours are being pursued. They will be presented in the next section.

\section{Three kinds of virtual tours}

Three different kinds of virtual tours are being pursued at the KATRIN experiment: guided video tours, a $360^{\circ} \mathrm{VR}$ panorama and the use of low-poly models. This variety allows to target audiences with different prerequisites and in different context. Key parameters of the three approaches are compared in table 1.

\subsection{Guided video tours}

In order create an online version of the typical tours given on site, a guided video tour was set up. In most cases, it starts with a slide-based introductory talk of about $20 \mathrm{~min}$ about neutrino physics, the KATRIN measurement principle and its setup. Then the experimental setup is shown with a self-made video, which is commented live and paused where necessary, leading to a $40 \mathrm{~min}$ tour based on a $20 \mathrm{~min}$ video. Starting at the source and moving along the whole beamline, the video extends the possibilities of an on-site tour with additional material: for example animations

Table 1: Properties of the virtual tours, targeting different audiences and occasions.

\begin{tabular}{lccc}
\hline & Video tour & $360^{\circ}$ VR panorama & Low-poly model \\
\hline $\begin{array}{l}\text { Language } \\
\text { Medium } \\
\text { Production }\end{array}$ & $\begin{array}{c}\text { English or German } \\
\text { zoom (video mode) } \\
\text { smartphone + blender }\end{array}$ & $\begin{array}{c}\text { German } \\
\text { web browser / VR headset } \\
\text { made by kubikfoto }\end{array}$ & $\begin{array}{c}\text { English or German } \\
\text { web browser } \\
\text { work in progress }\end{array}$ \\
\hline $\begin{array}{l}\text { Audience / occasion } \\
\text { Students }\end{array}$ & pupils & Master students & new collaborators \\
General public & yes & yes & at special interest \\
Evaluation reports & yes & no English version yet & yes \\
\hline
\end{tabular}


of the KATRIN measurement principle (which were created for the NaWik research discussed in section 4) and archive footage of the main spectrometer's construction and transport.

The tour video was cut in blender ${ }^{1}$. For the self-shot footage of the beamline, a Huawei Mate 20 was used ${ }^{2}$. Although the visual quality is no match for a professional camera setup and might be replaced in the future, the use of a smartphone offers several advantages:

- No costs. In combination with open source software for cutting, a smartphone video comes for free.

- Image stabilisation. Even without mechanical support like a gimbal, the image stabilisation software of the smartphone leads to reasonable results. Due to the narrow lab surroundings at the KATRIN beamline, mostly pan shots are used, which require no walking with the camera.

- Flexibility. A smartphone camara is continuously available and allows to record videos even on short notice when the measurement conditions allow access to otherwise restricted parts of the beamline. This also facilitates reshoots.

The introductory talk and tour video are both presented in zoom, where the screen sharing is optimised for videos and the sound sharing is activated. Each participant can ask questions at any time during the tour to create the immediate contact between visitors and scientists as on site. In one case of a large audience of aobut 100 people, the zoom session was streamed via YouTube and questions were collected and asked by a moderator in zoom.

Occasions for the video tour included the Karlsruhe Science Night ${ }^{3}$ in November 2020 and events organised by "Netzwerk Teilchenwelt" 4 . After one such event for pupils, teachers and the interested public, non-representative feedback was collected from nine persons: eight out of nine people felt that they obtained a good impression of the physical size of the experiment, while the video quality was perceived very good (five people) or good (four people).

\section{2 $360^{\circ}$ VR panorama}

The $360^{\circ}$ VR panorama offers a photorealistic view of the KATRIN experiment at five locations along the beamline. It can be used with a VR headset or as a browser application and was created by the company kubikfoto ${ }^{5}$. The panorama was developed in collaboration with the National Institute for Science Communication (NaWik), which also investigates on the reception of the VR panorama by laypersons and the knowledge transfer potential for students (see section 4).

The application consists of two subsequent parts, which can be started independently:

- Guided tour. In a live-action recording, the tour guide explains the measurement principle of the KATRIN experiment and details the mechanisms for ion blocking and removal in the transport section. The tour leads through all five locations and asks the user to adapt the source gas density, the high voltage of the main spectrometer and the voltage on the ion

\footnotetext{
${ }^{1}$ Homepage: https://www.blender.org/

2Product page: https://consumer . huawei .com/en/support/phones/mate20/

${ }^{3}$ Homepage: https://ndw-ka.de/

${ }^{4}$ Homepage: https://www.teilchenwelt.de/

${ }^{5}$ Homepage: https://www . kubikfoto.de/de/
} 
retention electrodes. The effect of these settings on the particles inside the beam tube can be observed with an "X-ray" view.

- Free exploration. Again, all five locations can be visited and also the "X-ray" view is still available. Instead of the tour guide, various scientists appear during the exploration to explain their work and the physics and technology of KATRIN. Furthermore, numerous info points allow the free inspection of additional pictures and videos, most of which are also used in the guided video tour.

\subsection{Low-poly model of the detector section}

An interactive 3D viewer of the beamline for web browsers is work in progress. The goal is to allow any user the free navigation around the beamline, hiding or unhiding of components and automatically zoom onto them. Detailed explanations on the main components should be given in a legend and labels with details of component parts shown in the viewport.

So far, a low-poly model of the detector section was created in blender and two different approaches are being pursued to realise the 3D viewer:

- Babylon.js ${ }^{6}$ is an open source software under the Apache 2.0 license, which offers a WebGL library for the visualisation of low-poly meshes in JavaScript. HTML and CSS are used to create the web page around the $3 \mathrm{D}$ viewport.

- Unity ${ }^{7}$ offers a complete game engine for the visualisation and animation of mesh models, based on C\# scripts. The result can be built as WebGL application, as well as a stand-alone application for PC or as Android app. Lately, funding was secured for a commercial license.

\section{Reception study by the National Institute for Science Communication (NaWik)}

The creation of virtual outreach tools about the KATRIN experiment at KIT is accompanied by research of the National Institute for Science Communication (NaWik). In the scope of the research project Science In Presentations (SIP), different forms of presentation in external science communication have been investigated since 2015. Research partners are the Department of Science Communication at the KIT and the NaWik. The project is funded by the Klaus Tschira Foundation.

Within the framework of this research project, among other things, a video series with four videos was produced, the content of which deals with the basics of the KATRIN experiment. The aim of this production was to investigate the influence of different forms of presentation in communicating scientific content, so the videos are almost identical in terms of content and language. The main difference is the form of presentation chosen in each case: a classic PowerPoint presentation is used in one video, an animation with a 3D perspective in another, a model is used for explanations in a third video, and on location in the fourth, similar to classic TV reports. The animations of the KATRIN measurement principle, which have been produced specifically for this research project, are also being used for the guided video tour (section 3.1) and the $360^{\circ} \mathrm{VR}$ panorama (section 3.2).

\footnotetext{
${ }^{6}$ Homepage: https://www.babylonjs.com/, GitHub: https://github.com/BabylonJS/Babylon.js
}

${ }^{7}$ Hhomepage: https://unity.com/ 


\section{Number of correct statements in pre- \& post-treatment (average)}

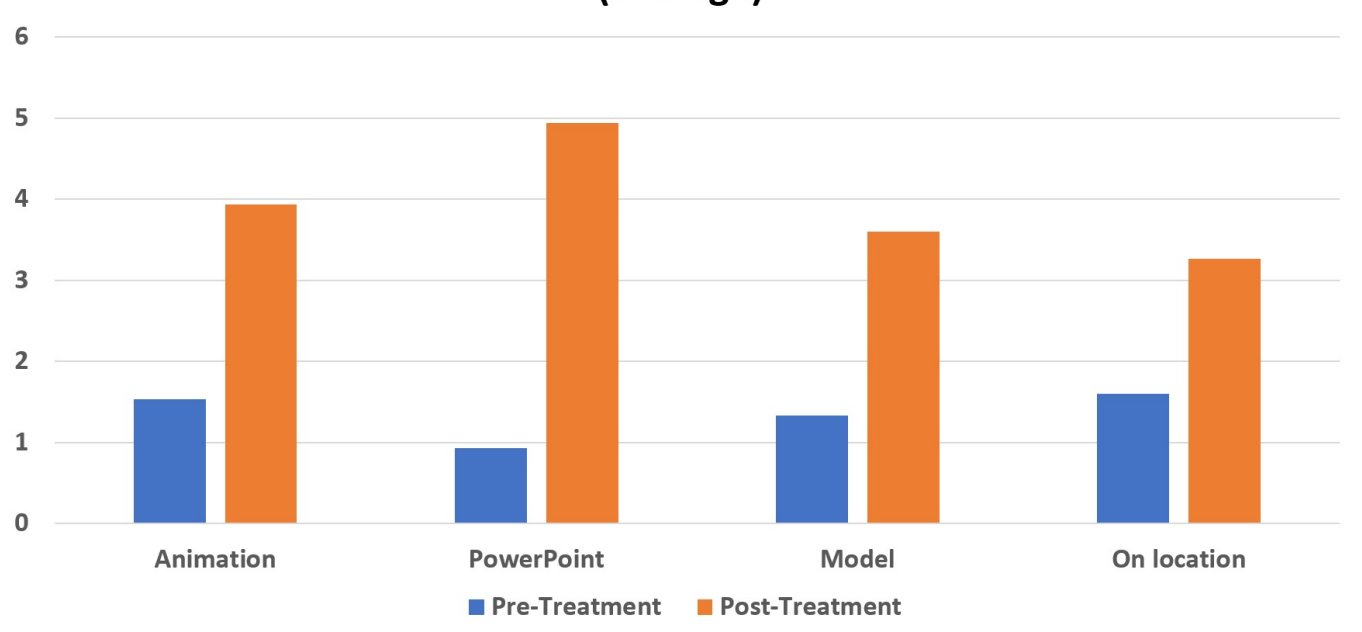

Figure 2: Knowledge transfer potential of virtual presentation forms. The study was performed with a total of 60 test persons.

The reception study with a total of 60 test persons uses a multi-method design in which eyetracking, guided interviews, questionnaires, postactional thinking aloud and the concept mapping method were combined to record structural knowledge. The evaluations have not yet been completed, but figure 2 already shows a result from the structural knowledge test: the average number of correct statements that the test subjects were able to make per variant pre- and post-treatment. The PowerPoint variant performed particularly well here.

As a follow-up to the four video formats, the NaWik co-produced the $360^{\circ} \mathrm{VR}$ panorama of the KATRIN beamline from section 3.2. This was also examined comparatively and the evaluations are still underway. Furthermore, an investigation of the $360^{\circ}$ VR environment in a teaching context with physics students is planned.

\section{Summary and conclusion}

The KATRIN experiment searches the absolute neutrino mass by measuring the tritium beta spectrum near its kinematic endpoint based on the MAC-E filter principle [1, 4]. In 2021, KATRIN achieved the first model-independent sub-eV neutrino mass limit of $m_{v}<0.8 \mathrm{eV} / \mathrm{c}^{2}$ (90\% C.L.) [6]. The 70 meter long KATRIN beamline poses many technological challenges, which had to be overcome in its almost two decades long history.

In order to present the KATRIN beamline to the students and the interested public, guided tours are frequently offered on site. Additionally, virtual tours have been developed due to multiple benefits: they allow permanent access to parts of the beamline which cannot be visited during the measurement operation; they allow to visualise the KATRIN beamline and dynamic particle 
processes inside; and this in turn offers an interactive and intuitive approach to the experiment for laypersons.

Owed to the large range of target audiences and occasions, three different kinds of virtual tours have been developed: guided video tours present the key aspects of the physics and technology in KATRIN, while allowing for immediate interaction and feedback between guide and audience; a $360^{\circ}$ VR panorama of five locations along the beamline including info points and optional guide can be explored freely at any time with a VR headset or in a web browser; and a 3D viewer of a low-poly model of the detector section for free exploration in web browsers is work in progress.

The KATRIN outreach projects at KIT profit from the collaboration with the National Institute for Science Communication (NaWik), which funded animations about the KATRIN measurement principle and co-funded the $360^{\circ} \mathrm{VR}$ panorama. The NaWik has also conducted a study about the reception of the $360^{\circ} \mathrm{VR}$ panorama and is currently investigating its knowledge transfer potential for use in teaching in the Master curriculum.

\section{Acknowledgements}

Supported by BMBF (Ø05A20VK3), the Helmholtz Association, the Klaus Tschira Foundation, the KIT centre KCETA, and the Excellence Strategy of the German Federal and State Governments. Further, we would like to thank Katrin Link and the organisers of the week of particle physics from the "Netzwerk Teilchenwelt".

\section{References}

[1] J. Angrik et al. (KATRIN Collaboration), KATRIN Design Report 2004, Wissenschaftliche Berichte FZKA 7090, http://bibliothek.fzk.de/zb/berichte/FZKA7090.pdf

[2] V. M. Lobashev and P. E. Spivak, A method for measuring the electron antineutrino rest mass, Nuclear Instruments and Methods in Physics Research Section A: Accelerators, Spectrometers, Detectors and Associated Equipment 240.2 (1985): 305-310.

[3] A. Picard et al., A solenoid retarding spectrometer with high resolution and transmission for keV electrons, Nuclear Instruments and Methods in Physics Research Section B: Beam Interactions with Materials and Atoms 63.3 (1992): 345-358.

[4] M. Aker et al. (KATRIN Collaboration), The Design, Construction, and Commissioning of the KATRIN Experiment, arXiv preprint arXiv:2103.04755 (2021).

[5] M. Aker et al. (KATRIN Collaboration), Improved upper limit on the neutrino mass from a direct kinematic method by KATRIN, Physical review letters 123.22 (2019): 221802.

[6] M. Aker et al. (KATRIN Collaboration), First direct neutrino-mass measurement with sub-eV sensitivity, arXiv preprint arXiv:2105.08533 (2021). 